\title{
A Practitioner Tool for Developing and Measuring the Results of Interventions
}

\author{
Sharon Zivkovic \\ University of South Australia
}

It is not uncommon for practitioners to attempt to address a wicked problem by developing an intervention that only addresses a few of the problem's underpinning causal factors and not the wicked problem in its entirety. To address the whole wicked problem, practitioners need to work through collaborative networks to transition ecosystems of initiatives to a new improved state, or develop interventions that create systems change by influencing others to take action. This paper examines a matrix tool that was created to assist practitioners to develop and evaluate interventions that address simple, complicated, complex and wicked problems. The tool highlights that the type of intervention and the type of measurement that is most appropriate will depend on the scope and the causal logic of the intervention. The four quadrants of the matrix are: basic services, integrated services, social movements and solution ecosystems. To demonstrate how this tool can assist practitioners to develop and measure the results of initiatives, the paper refers to two Australian case studies.

Keywords: wicked problems complex measurement tool interventions

\section{INTRODUCTION}

Developing an intervention is the traditional response to addressing societal problems. Many of these interventions are underpinned by a program logic that specifies the intervention's cause and effect relationships (WK Kellogg Foundation, 2004). While a program logic approach is well suited to addressing simple and complicated problems, as they can be understood through cause and effect logic (Westley et al., 2007), it is not appropriate for addressing wicked problems, as they have a multitude of underpinning and intertwined causal factors that do not follow an easy to understand results chain (Australian Public Service Commission 2007). To address wicked problems in their entirety, instead of taking a program logic approach, practitioners need to either transition ecosystems of initiatives to a new and improved state by working through collaborative networks (Lichtenstein and Plowman, 2009), or they need to develop interventions that influence many others to take action to address the problem (Martin and Osberg, 2007).

This paper describes and demonstrates a tool—an Intervention Development and Results Measurement (IDRM) Matrix - that was created to assist practitioners to develop interventions that are appropriate for the type of problem that they are addressing, and to determine the most suitable approach for measuring the performance of that intervention. Four types of problems are included in the tool: simple, complicated, complex and wicked problems. The IDRM Matrix suggests that when developing an intervention, or measuring the effect of an intervention, the approach taken will depend on the scope and the causal logic of the intervention. These two variables, scope and causal logic, are the two axis of the IDRM Matrix. The 
four quadrants of the IDRM Matrix represent the four intervention types that are illuminated using the tool: basic services, integrated services, social movements and solution ecosystems.

The IDRM Matrix was developed by adapting Ebrahim and Rangan's (2010) contingency framework for measuring social performance to include problem types. While the IDRM Matrix was originally used to aid practitioners in the development of innovations, it has also been used by practitioners for results measurement. It was from using the IDRM Matrix for results measurement that the need to incorporate macro environmental factors when addressing complex and wicked problems was suggested. This feature has since been incorporated into the IDRM Matrix.

This paper is structured as follows. After differentiating problem types, the paper outlines how best to address each of the identified problem types by referring to the IDM Matrix. Then for each of these approaches, the IDRM Matrix is used to suggest how to best measure the results for each intervention approach. Finally, the paper describes how to use the IDRM Matrix in practice for the development of interventions and the measurement of an intervention's results by referring to two Australian case studies. The first case study is the use of the matrix by Community Capacity Builders to design its new Program for Social Entrepreneurs. The second case study is the use of the IDRM matrix by Wicked Lab to evaluate the pilot of its Complex Systems Leadership Program.

\section{PROBLEM TYPES}

According to Snowden and Boone (2007), the first step in addressing any problem should be to identify the problem's type, as different types of problems need to be addressed in different ways. Typologies used for analysing problems include Westley et al.'s (2007) classification of problems as simple, complicated and complex; and Rittel and Webber's (1973) differentiation between tame and wicked problems.

\section{Simple Problems}

Simple problems have clear relationships between cause and effect that enable a right answer to be determined in advance, a standardised best practice solution to be developed, and command and control management to be effective (Westley et al., 2007; Snowden and Boone, 2007). Given these characteristics, addressing simple problems has been likened to following a recipe when baking a cake (Westley et al., 2007). Procedures and best practice manuals are often written for addressing simple problems. It is easy to replicate interventions that address simple problems: you just need to follow the recipe.

An example of addressing a simple problem would be an immunisation procedure. A linear program logic, like a recipe, can be developed for the procedure: there would be inputs such as the injection and the vaccine, the activity of inoculating people would be carried out, which would produce so many inoculated people. The relationships between inputs and outputs would be linear: the size of the outputs (people inoculated) would be proportional to the size of the inputs (the inoculations).

\section{Complicated Problems}

Like simple problems, complicated problems have clear relationships between cause and effect, the relationship between inputs and outputs is linear, and solutions can be determined in advance (Westley et al., 2007; Snowden and Boone, 2007). Complicated problems differ from simple problems though in that they have many more components compared to simple problems and because of their many parts they can have a number of right answers (Westley et al., 2007; Snowden and Boone, 2007). Since they can have a number of right answers, they are addressed through good practice rather than best practice solutions (Snowden, 2002).

An example that is often used for addressing a complicated problem is sending a rocket to the moon (Westley et al., 2007). A blueprint for the rocket would be developed that specifies the separate rocket component parts and the relationships by which the parts need to be assembled. There would not be the one best practice rocket design, there would be good practice rocket designs, as the many parts could be arranged differently. It would be easy to replicate the rocket design: you would just follow the blueprint. Following the blueprint would ensure critical success factors are included each time a rocket is sent to the 
moon. With the relationship between inputs and outputs being linear, if you have so many rocket parts you would be able to build one rocket. If you have double the amount of parts you would be able to build two rockets.

An example of addressing a complicated problem that is more relevant to addressing social problems would be how do you assist people to find employment. A theory of change could be developed for an employment program that specifies its many skills development, employment placement and postplacement support parts, and its assumptions about how the parts would work together. It would not be the one best practice employment program, but if designed and implemented well it could be a good practice employment program. To replicate the employment program, you would follow the program's theory of change.

\section{Complex Problems}

Compared to simple and complicated problems, complex problems are a very different type of problem. They do not have clear relationships between cause and effect. Instead they have a multitude of intertwined and interconnected causes: they have many interdependent parts (Westley et al., 2007; Snowden and Boone, 2007). Because of this multicausality and interdependency, the parts of the problem change each other and the problem is constantly adapting (Westley et al., 2007; Snowden and Boone, 2007). Complex problems are more than the sum of their parts (Snowden and Boone, 2007): there is an essence in the interacting relationships between the people, experiences and moments in time that constitute the problem (Westley et al., 2007).

Given these characteristics, complex problems are unpredictable, they do not have a right answer, and relationships between cause and effect can only be determined in retrospect (Snowden and Boone, 2007). Solutions to complex problems are therefore not replicable as solutions are only appropriate for the specific issue, in the specific context, at the specific moment in time in which the solution was created. Finding a right answer is therefore not the focus when addressing complex problems.

An example of addressing a complex problem is how do you raise a child (Westley et al., 2007): every child is different, following a recipe or blueprint generally does not work and is often detrimental, and raising one child successfully does not guarantee success with raising a second child. There are many causal, intertwined and interdependent factors that influence how successfully you can raise a child.

For complex problems, like raising a child, the whole is greater than the sum of the parts: raising a child is more than the sum of the child's family life, friends, education, socio-economic status etc. It is about the intertwined and interdependent nature of these underpinning causal factors. To address complex problems the focus needs to be on the whole system: the whole ecosystem of the problem's intertwined and interdependent underpinning causal factors and the whole ecosystem of interventions that are addressing any of these underpinning causal factors.

\section{Wicked Problems}

Rittel and Webber (1973) differentiate wicked problems from tame problems. Wicked problems are considered "wicked" in the sense that they are highly resistant to resolution (Australian Public Service Commission, 2007). Tame problems are more easily resolved as they have a clear mission, all the information required for understanding and solving them is available, and it is easy to determine if the problem has been solved or not (Rittel and Webber, 1973). Rittel and Webber's (1973) tame problem classification aligns with Westley et al.'s (2007) simple and complicated problem types.

Wicked problems are society's most pressing social problems (Rittel and Webber, 1973). They are the complex social policy problems that societies face that cannot be successfully addressed with traditional linear, analytical approaches (Rittel and Webber). Wicked problems have the same properties as Westley et al.'s (2007) complex problems, but in addition they are the focus of government policy. Examples of wicked problems include: terrorism, environmental degradation, poverty (Krawchulk 2008), food insecurity (Zivkovic, 2017), climate change, obesity, indigenous disadvantage (Australian Public Service Commission 2007) and active citizenship (Day 1997). 
While the range of problems that are classified as being "wicked" are quite diverse, they all share the same characteristics. These include: they have multiple causes, they have many interdependencies, different stakeholders have a different understanding of what the problem is and therefore different stakeholders have conflicting goals, they have no clear solution, attempts to address them often leads to unforeseen consequences due to their multi-causality and interdependency, they adapt: because of the interdependencies changes to one part of the problem can have unforeseen consequences for other parts of the problem, and they are context specific (Australian Public Service Commission, 2007). The diagram of the Obesity System Map, shown if Figure 1, illuminates these characteristics for the wicked problem of obesity.

\section{FIGURE 1 OBESITY SYSTEM MAP}

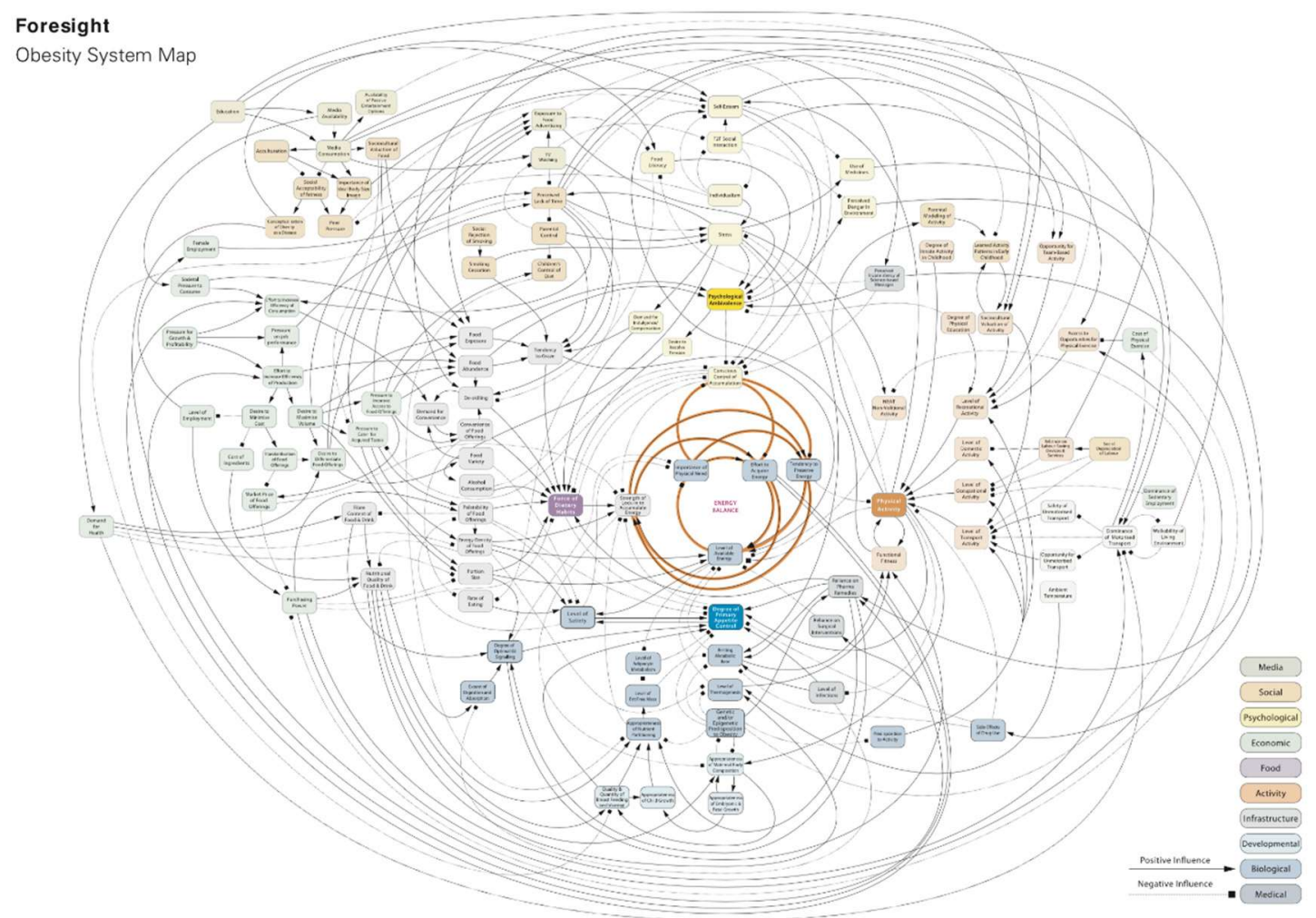

(BUTLAND ET AL., 2007)

The Obesity System Map diagram highlights the numerous causal factors that influence obesity and the multitude of interdependencies between these causal factors. Given these characteristics it is easy to see: why it is not uncommon for different stakeholders to have a different understanding of what causes obesity, why these different stakeholders would have different goals for addressing obesity, and why obesity does not have a clear solution. The feedback loops between the causal factors underpinning obesity suggest the reason for obesity being adaptive, and why attempts to address obesity through interventions focusing on one part of the wicked problem can lead to unforeseen consequences in other parts of the problem. It can also be seen by looking at the Obesity System Map, why the problem of obesity would be different in different contexts: the causal nodes and feedback loops would have different values in different communities. 


\section{ADDRESSING PROBLEM TYPES}

When designing interventions to address a problem the intervention approach needs to be appropriate for the type of problem that is being addressed. For simple problems that have few component parts and clear relationships between cause and effect, a logic model can be used. Logic models show the cause-andeffect relationships between the activities undertaken and the results that it is hoped are achieved from the activities (Wei-Skillern et al., 2007).

For complicated problems that have clear relationships between cause and effect, but more component parts than simple problems, a theory of change can be used. Theories of change contain the underlying assumptions about how and why an initiative will achieve the expected results, the outcomes it is expected to achieve over time, and the connection between its strategies and outcomes (Gutierrez and Tasse, 2007).

For problems that do not have clear relationships between cause and effect, such as complex and wicked problems, the Law of Requisite Variety provides insights for how to address them. The Law of Requisite Variety states that "only variety can destroy variety" (Ashby, 1956) which suggests that to control the variety of a system, such as all of the causal factors and interdependencies underpinning a complex or wicked problem, the approach for addressing the problem needs to have as much variety as the problem (Batty, 2007).

Social movements and solution ecosystems have as much variety as complex and wicked problems. Social movements focus on a specific change agenda. They are "a broad set of sustained organizational efforts to change the structure of society or the distribution of society's resources" (Coglianese, 2001, p. 85). Solution ecosystems focus on integrated interventions that are complimentary. They consist of all of the interventions in a geographical community that are addressing any of the underpinning causal factors of the targeted wicked problem, and all the organisations that are partnering on those interventions (Eggers and Muoio, 2015).

There are two key differentiators that need to be considered when designing interventions for the different problem types: the intervention's scope and the causal logic accepted by the intervention. An intervention's scope depicts how comprehensive an intervention's focus is and how integrated an intervention's activities are. Interventions that have a specific focus offer a single, highly specific service or agenda. Interventions that have an integrated complementary focus have an integrated set of complementary activities, services or initiatives.

An intervention's causal logic is the relationship between cause and effect for the intervention. Interventions that have a linear and understood causal logic have clear relationships between cause and effect, these relationships between cause and effect can be understood, and the relationships between inputs and outputs is linear which means the size of the output, such as the number of people inoculated, is proportional to the size of the inputs, such as the number of inoculations. Interventions that have a multicausal, intertwined and not well understood causal logic do not have clear relationships between cause and effect, the relationships between cause and effect are not well understood, their relationships between inputs and outputs are not linear and they generally have tipping points: they need to reach a threshold before significant change occurs.

Figure 2 shows the IDRM Matrix which is based on these two key differentiators. The Intervention Scope is shown on the horizontal axis, and the Causal Logic is on the vertical axis. The four quadrants in the matrix provide guidance on how to address different types of problems with interventions. 


\section{FIGURE 2 \\ INTERVENTION DEVELOPMENT AND RESULTS MEASUREMENT MATRIX}

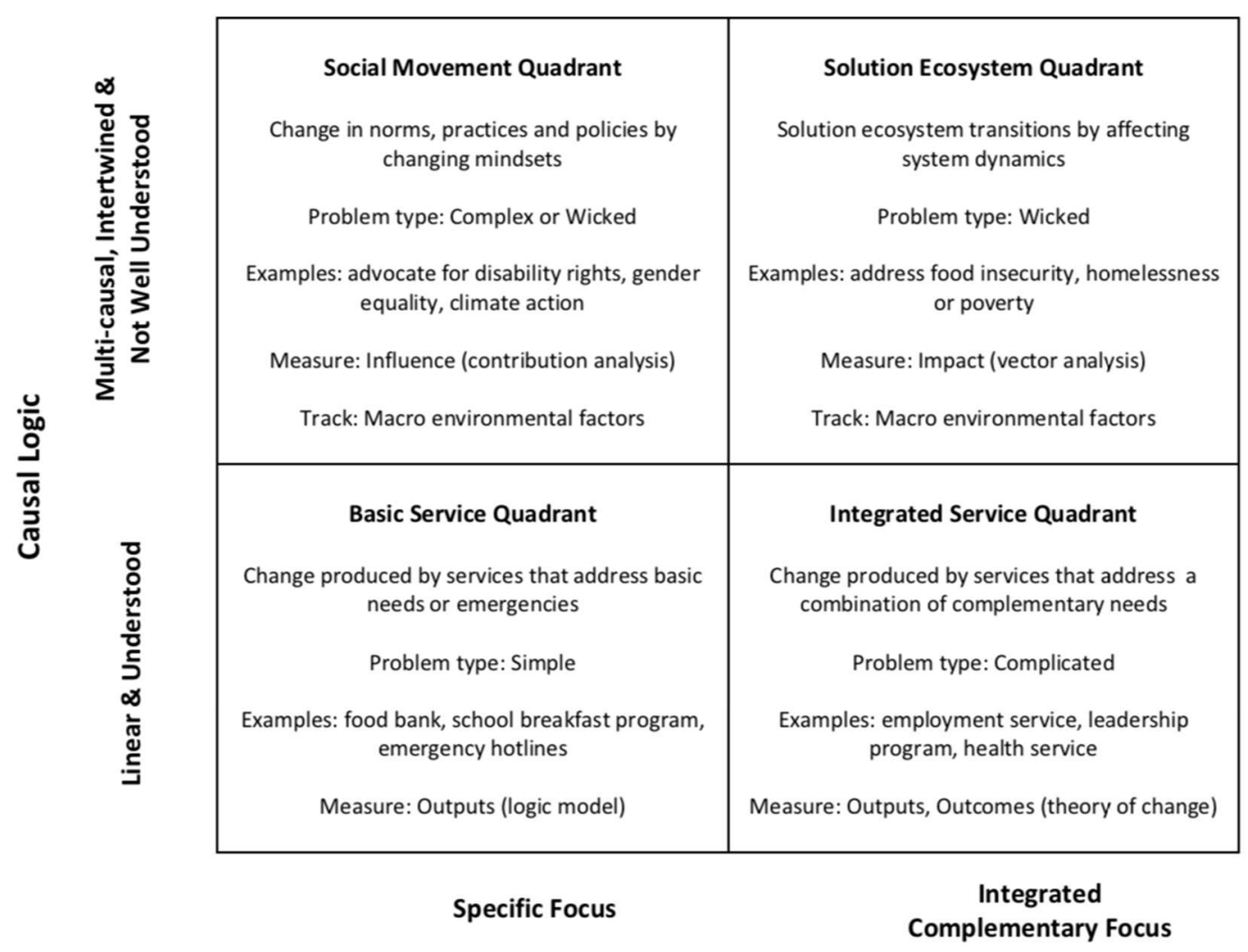

Intervention Scope

\section{Simple Problems}

The Basic Service Quadrant in the IDRM Matrix suggests how to address simple problems. The interventions that address simple problems have a very narrow and specific focus and are driven by a causal logic that is linear and well understood. For these interventions, given you can easily understand the relationships between cause and effect, a logic model can be developed to address the problem. Examples of interventions in the Basic Services Quadrant include food banks, school breakfast programs, and emergency services such as emergency hotlines. Such services aim to meet immediate and very specific pressing needs.

\section{Complicated Problems}

The Integrated Service Quadrant in the IDRM Matrix focuses on addressing complicated problems. Interventions in this quadrant have a much broader scope than interventions in the Basic Service Results quadrant: they focus on a number of integrated and complementary activities and address a combination of complementary needs. Like simple problems, complicated problems also follow a causal logic that is linear and well understood, but because they have many more parts that can be arranged in different ways assumptions are made about the optimum way to arrange the parts for the intervention. To incorporate these assumptions, a theory of change can be developed for the intervention. Examples of interventions that often address a number of complementary needs include employment services, leadership programs and health services. 


\section{Complex and Wicked Problems}

Interventions in the Social Movement and the Solution Ecosystem Quadrants of the IDRM Matrix work with a multi-causal logic consisting of intertwined and not well understood causal logic. The Social Movement Quadrant focuses on addressing complex and wicked problems by having a specific agenda to advocate for and encourage systems change. If the complex problem that is the focus of the advocacy and encouragement is also the subject of government policy it would generally be referred to as a wicked problem (Rittel and Webber, 1973).

Instead of interventions in the Social Movement Quadrant producing integrated systems change themselves, they influence others to change norms, practices and policies (Martin and Osberg, 2007). An intervention in this quadrant would be one of a number of interventions that are advocating for and encouraging the change, and there would also be contextual factors in the macroenvironment that are contributing towards the change. Examples of interventions in the Social Movement Quadrant include interventions that advocate for and encourage disability rights, gender equality and climate action.

The Solution Ecosystem Quadrant in the IDRM Matrix focuses on addressing wicked problems by affecting the dynamics of solution ecosystems so that they can transition to a new way of working that has increased system functioning and performance. Solution ecosystems address the multiple intertwined causal factors underpinning wicked problems. They do this by increasing the coherent action amongst all of the initiatives and organisations in an ecosystem that are addressing any of a wicked problem's intertwined causal factors. Examples of interventions in the Solution Ecosystem Quadrant include solution ecosystems that address food insecurity, homelessness and poverty.

\section{MEASURING RESULTS}

According to Snowden (2017), measurement systems need to be created "that are authentic to the thing being measured". Given the diverse characteristics of the various problem types, it is logical to expect that the results of interventions that address different types of problems would be measured differently.

\section{Simple Problems}

For interventions in the Basic Services Quadrant, it is quite easy to measure the outputs from their activities, such as how many people have been fed or how many phone calls have been taken. However, if attempts are made to measure outcomes or impacts, complicated, time consuming and expensive measurement approaches would need to be applied. Therefore, measuring outputs is appropriate for interventions in the basic services quadrant. Measuring outcomes or impacts, such as the impact of a food bank service on the community's food security or tracking what people do with the information they receive from the phone call of an emergency hotline, would be beyond the aim, scope and control of basic service interventions.

\section{Complicated Problems}

Because interventions in the Integrated Service Quadrant address a combination of related needs, they have greater control over achieving lasting benefits for people than the services in the Basic Service Quadrant. For example, an employment service has more control over achieving employment outcomes for people by integrating a number of activities such as skills development, employment placement and postplacement support. Given this greater control, in addition to measuring their outputs such as number of people trained, number of people placed in employment and number of post-placement support visits, interventions in the Integrated Service Quadrant can measure outcomes such as the number of people still employed after one year.

While integrated services have more control over achieving outcomes because they address a combination of related needs, in reality they still would not have complete control over achieving outcomes as there would be other factors outside of their control that could affect the achievement of outcomes. Despite this limitation, measuring outcomes when addressing complicated problems is still undertaken. This is because, as highlighted by Forss and Schwartz (2011, p. 16), "Even though, in terms of ontology 
and epistemology, everything is complex, we can often make practical progress by realizing that some tasks are better understood and dealt with as not so complex".

Given this limitation, when addressing complicated problems, results measurement needs to be viewed as a continual learning and improvement process: the assumptions in the intervention's theory of change, which describe how the integrated activities work together as a coherent intervention to address the problem, need to be continually tested in practice to identify other factors that could affect outcomes. Taking this approach, the object of analysis for results measurement when addressing complicated problems is the intervention itself, and not the performance measurement of the individual, team or organisation that is implementing the initiative: the objective is learning and improving the intervention. The measurement of outcomes when addressing complicated problems is undertaken because "funders need to know whether their funds are making a difference in solving societal problems and... [initiative] managers desire to gain awareness regarding the outcomes and impacts of their activities" (Costa and Pesci, 2016, p. 100).

There are a number of meanings that have been attributed to the word "impact" when discussing the impacts of activities. These include: changes to people's lives as a result of an intervention that are significant or lasting, the meaning attributed to the impact component of a logic model which is systems change, and the outcomes of an intervention after what would have occurred without the intervention is removed from the equation (Rangan et al., 2011). For the Integrated Service Quadrant of the IDRM Matrix, it is the outcomes of an intervention that is the correct meaning.

\section{Complex and Wicked Problems}

Measuring results when addressing complex and wicked problems, is very different than measuring results for simple and complicated problems. According to Snowden (2017), instead of trying to manage a desired future state, when addressing complex and wicked problems the focus needs to be on managing "the evolutionary potential of the situated present". How to measure results when addressing complex and wicked problems also depends on if the intervention: advocates for systems change in complex systems, or achieves systems change by addressing the system dynamics of a complex system.

Advocating for systems change in complex systems is the focus of the Social Movement Quadrant of the IDRM Matrix. For the Social Movement Quadrant, if there is systems change, the intervention would be one of a number of interventions and macroenvironmental factors that are responsible for the change. Therefore, while the system change cannot solely be attributed to the intervention, the intervention could have had an influence in creating the systems change. Interventions in the Social Movement Quadrant can measure their influence in changing norms, practices and policies by undertaking a contribution analysis. Contribution analysis "provides evidence and a line of reasoning from which we can draw a plausible conclusion that, within some level of confidence, the program has made an important contribution to the documented results" (Better Evaluation, n.d.). Interventions in the Social Movement Quadrant can also track changes in the macroenvironmental factors that impact on the complex problem.

Achieving systems change by addressing the system dynamics of a complex system is the focus of the Solution Ecosystem Quadrant of the IDRM Matrix. The Solution Ecosystem Quadrant focuses on measuring impact. The meaning attributed to the word "impact" in the Solution Ecosystem Quadrant is the impact component of a logic model (Rangan et al., 2011) as solution ecosystems are concerned with producing systems change through transitions. The measurement of this systems change is undertaken through vector measurement: measuring for a solution ecosystem "the direction and speed of travel from the present" (Snowden, 2016).

For transitions, the direction of travel to a new state is well understood, as highlighted in the following quote: "Emergence [of a new state] follows a now well-understood route ......In other words, a new order appears if forces at play exert tension on the system; a small change, if amplified, leads to a transformative process which, fuelled with the new imported resources and positively reinforcing forces, leads to a new equilibrium" (Thietart and Forgues, 2011).

The stages of this well understood direction of travel are shown in Figure 3. Panel 1 in Figure 3 represents the current working relationships and ways of working for a solution ecosystem. At Panel 2 the solution ecosystem is disrupted and a disequilibrium state is created. At Panel 3 the new way of working is 
amplified to stop the solution ecosystem going back to the old way of working. At Panel 4 the solution ecosystem is assisted to self-organise around the new way of working: a way of working that is more coherent, and has increased system functioning and performance for addressing wicked problems. At Panel 5 the solution ecosystem is stabilised around the new way of working: the new state.

FIGURE 3

STAGES OF DIRECTION OF TRAVEL

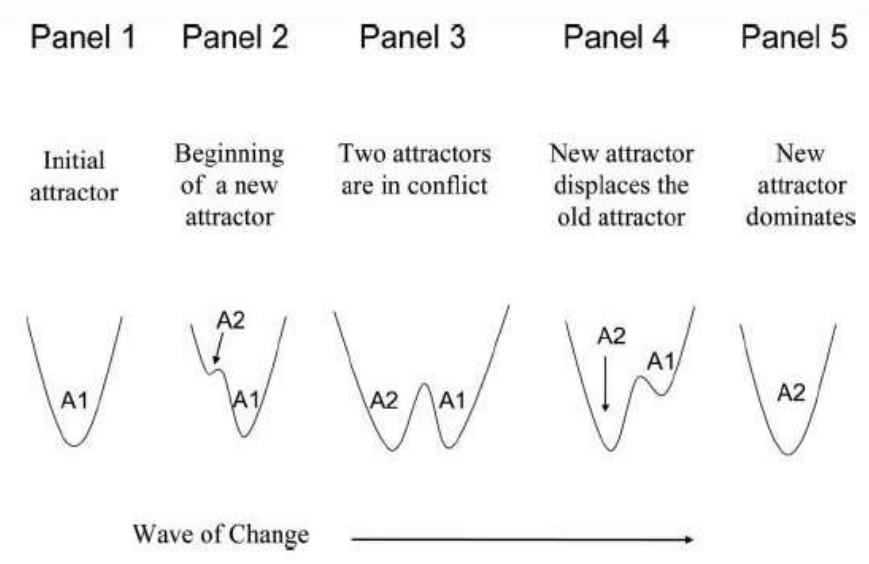

(GOLDSTEIN, J., HAZY, J. K., \& SILBERSTANG, J., 2010)

\section{CASE STUDY: USING THE IDRM MATRIX TO DEVELOP INTERVENTIONS}

Community Capacity Builders has used the IDRM Matrix to help it think through the development of its new Program for Social Entrepreneurs. Community Capacity Builders Program for Social Entrepreneurs consists of four units: Understanding Social Entrepreneurship, Mapping Problem \& Solution Ecosystems, Developing Initiatives, and Developing Social Enterprises.

While this program supports social entrepreneurs to develop interventions that address complicated problems, it also increases participants awareness that their intervention can contribute toward addressing a wicked problem if it is part of a solution ecosystem that is addressing their complicated problem's overarching wicked problem. The Community Capacity Builders program therefore bridges the Integrated Service Quadrant and the Solution Ecosystem Quadrant.

In Unit 2 of the program there is a link to the Solution Ecosystem Quadrant. During this unit participants research and map the problem and solution ecosystems for the problem that they are addressing. It is during Unit 2 that it is highlighted to participants that the initiative that they choose to develop to address a complicated problem can be a part of a solution ecosystem that is addressing an overarching wicked problem.

In Unit 3 of the program there is a link to the Integrated Service Quadrant. During this unit participants determine where to intervene in a solution ecosystem, and develop a theory of change for the intervention that they create. The program states that "Theories of change contain the underlying assumptions about how and why an initiative will achieve its desired results and provide a roadmap that summarises the steps that need to be taken to achieve the desired results" (Community Capacity Builders, n.d.).

\section{CASE STUDY: USING THE IDRM MATRIX TO MEASURE THE RESULTS OF INTERVENTIONS}

Wicked Lab used the IDRM Matrix to inform the evaluation of its Complex Systems Leadership Program's pilot. This program supports a systemic innovation approach to addressing wicked problems. Systemic innovation is defined as: "a set of interconnected innovations, where each is dependent on the 
other, with innovation both in the parts of the system and in the ways that they interact" (Davies, et al., 2012, p. 4).

The program consists of three units of study which are undertaken online during a six-month period. Unit 1 focuses on participants understanding the characteristics of wicked problems and why a complexity approach is required to address them. In Unit 2 participants gain an understanding of initiative characteristics that assist communities to strengthen their adaptive dynamics and undertake transitions, and in Unit 3 they gain an understanding of initiative characteristics that assist governments to support transition approaches. Monthly online group sessions are held during the program to provide participants with the opportunity to ask questions and share experiences.

During each of the program's units, participants use Wicked Lab's online Tool for Systemic Change to address a wicked problem of their choice in a geographical community of their choice. In Unit 1, participants define the boundary of their solution ecosystem: the geographical boundary and the wicked problem, and enter into the software all of the initiatives within that geographical boundary that are addressing any of the underpinning causal factors of their targeted wicked problem. In Unit 2, for each of the initiatives that were entered into the software in Unit 1, participants identify if the initiative has any of the initiative characteristics that assist communities to transition to a new state that has increased coherence and performance. During Unit 3 participants identify if any of the initiatives have initiative characteristics that strengthen the interface between community and government systems.

Like Community Capacity Builders Program for Social Entrepreneurs, Wicked Lab's Complex Systems Leadership Program bridges the Integrated Service Quadrant and the Solution Ecosystem Quadrant of the IDRM Matrix. The Complex Systems Leadership Program aligns to the Integrated Service Quadrant as it addresses a complicated problem with integrated and complimentary activities. How to provide participants with the knowledge and skills required to address wicked problems is the complicated problem. The program's integrated and complementary activities for developing the required knowledge and skills include a diverse range of online learning materials, monthly group catch-up sessions, a project-based learning pedagogy and an online tool. As highlighted in the IDRM Matrix, because of its characteristics, it is appropriate to measure if participants have achieved the Complex Systems Leadership Program's learning outcomes.

Wicked Lab's Complex Systems Leadership Program and Tool for Systemic Change have been purposefully designed to assist communities and governments to take a solution ecosystem approach when addressing wicked problems which aligns to the Solution Ecosystem Quadrant of the IDRM Matrix. Participants in the program were able to measure systems change—impact—by using the Tool for Systemic Change's Transition Card Activity Report. This report provides a vector analysis: it measures 'the direction and speed of travel from the present' (Snowden, 2016) for all of the initiatives in a solution ecosystem.

\section{CONCLUSION}

This paper has highlighted that practitioners need to use different approaches to develop and evaluate interventions when addressing simple, complicated, complex and wicked problems. To assist practitioner to make intervention development and evaluation decisions, this paper has introduced an Intervention Development and Results Measurement (IDRM) Matrix.

This Matrix highlights that the type of intervention, and the type of measurement that is most appropriate, will depend on the scope and the causal logic of the intervention. These variables form the IDRM Matrix's horizontal and vertical axis which results in the four quadrants of: basic services, integrated services, social movements and solution ecosystems.

Two case studies have been discussed to highlight how the IDRM Matrix can be used in practice. Community Capacity Builders used the IDRM Matrix to help it think through the development of its Program for Social Entrepreneurs and Wicked Lab used the Matrix to help determine the results of its Complex Systems Leadership Program.

Both of these case studies bridged the Integrated Service Quadrant and the Solution Ecosystem Quadrant of the IDRM Matrix. This finding raises important questions. While interventions that address 
complicated problems cannot of themselves address wicked problems, effective interventions that address complicated problems could be a requirement for strengthening the solution ecosystems that are required to tackle wicked problems. It would be fruitful to pursue further research to investigate this finding.

\section{REFERENCES}

Ashby, W. R. (1956). An introduction to cybernetic. Chapman \& Hall, London.

Australian Public Service Commission. (2007). Tackling Wicked Problems: A Public Policy Perspective. Canberra.

Batty, M. (2007). Complexity in city systems: Understanding, evolution, and design. In E. Silva \& G. De Roo (Eds.), A Planner's Encounter with Complexity (pp. 99-122). Ashgate: Aldershot.

BetterEvaluation. (n.d.). Approaches: Contribution analysis. Retrieved from http://betterevaluation.org/plan/approach/contribution_analysis

Butland, B., Jebb, S., Kopelman, P., McPherson, K., Thomas, S., Mardell, J., \& Parry, V. (2007).

Tackling obesities: Future choices-project report. London: Department of Innovation, Universities and Skills.

Coglianese, C. (2001). Social movements, law, and society: The institutionalization of the environmental movement. University of Pennsylvania Law Review, 150(1), 85-118.

Community Capacity Builders. (n.d.). Program for Social Entrepreneurs: A Complex Systems Approach. Retrieved from https://www.communitycapacity.com.au/social-entrepreneurs.html

Costa, E., \& Pesci, C. (2016). Social impact measurement: Why do stakeholders matter? Sustainability Accounting, Management and Policy Journal, 7(1), 99-124.

Davies, A., Mulgan, G., Norman, W., Pulford, L., Patrick, R., \& Simon, J. (2012). Systemic Innovation. Social Innovation Europe. Retrieved from http://www.socialinnovationeurope.eu/sites/default/files/sites/default/files/SIE\%20Systemic\%20I nnovation\%20Report\%20-\%20December\%202012_1.pdf

Day, D. (1997). Citizen participation in the planning process: An essentially contested concept? Journal of Planning Literature, 11(3), 421-434.

Ebrahim, A., \& Rangan, V.K. (2010). The limits of nonprofit impact: A contingency framework for measuring social performance. Harvard Business School General Management Unit Working Paper (10-099).

Eggers, W., \& Muoio, E. (2015). Wicked Opportunities. In Deloitte Consulting LLP (Eds.), Business ecosystems come of age (pp. 31-42). Westlake, TX: Deloitte University Press.

Forss, K., \& Schwartz, R. (2011). Introduction. In K. Forss, M. Marra, \& R. Schwartz (Eds.), Evaluating the complex: Attribution, contribution, and beyond (pp. 1-32). New Brunswick: Transaction Publishers.

Goldstein, J., Hazy, J.K., \& Silberstang, J. (2010). A complexity science model of social innovation in social enterprise. Journal of Social Entrepreneurship, 1(1), 101-125.

Gutierrez, M., \& Tasse, T. (2007). Leading with Theory: Using a Theory of Change Approach for Leadership Development Evaluations. In K.M. Hannum, J.W. Martineau, \& C. Reinelt (Eds.), The Handbook of Leadership Development Evaluation (pp. 48-70). San Francisco: Jossey-Bass.

Krawchulk, F.T. (2008). Collaborative Strategic Planning and Action: A New Approach. Parameters, 38(2), 67-78.

Lichtenstein, B.B., \& Plowman, D.A. (2009). The leadership of emergence: A complex systems leadership theory of emergence at successive organizational levels. The Leadership Quarterly, 20(4), 617-630.

Martin, R.L., \& Osberg, S. (2007, Spring). Social entrepreneurship: The Case for Definition. Stanford Social Innovation Review, pp. 28-39.

Rangan, K.V., Appleby, S., \& Moon, L. (2011). The Promise of Impact Investing. Harvard Business School Background Note, (512-045). 
Rittel, H.W.J., \& Webber, M.M. (1973). Dilemmas in a general theory of planning. Policy Sciences, 4, $155-169$.

Snowden, D. (2002). Complex Acts of Knowing: Paradox and Descriptive Self Awareness. Journal of Knowledge Management, 6(2), 100-111.

Snowden, D. (2016). The banality of measurement. Retrieved from https://cognitive-edge.com/blog/thebanality-of-measurement/

Snowden, D. (2017). Systems thinking \& complexity. Retrieved from https://cognitiveedge.com/blog/systems-thinking-complexity/

Snowden, D.J., \& Boone, M.E. (2007). A leader's framework for decision making. Harvard Business Review, 85(11), 69-76.

Thietart, R., \& Forgues, B (2011). Complexity Science and Organisation. In P. Allen, S. Maguire, \& B McKelvey (Eds.), The SAGE Handbook of Complexity and Management (pp. 53-64). London: Sage Publications.

W.K. Kellogg Foundation. (2004). Logic Model Development Guide. Retrieved from https://www.wkkf.org/resource-directory/resources/2004/01/logic-model-development-guide

Wei-Skillern, J., Austin, J., Leonard, H., \& Stevenson, H. (2007). Entrepreneurship in the Social Sector. London: Sage Publications.

Westley, F., Zimmerman, B., \& Patton, M.Q. (2007). Getting to Maybe: How the World Is Changed. Toronto: Vintage Canada.

Zivkovic, S. (2017). Addressing food insecurity: A systemic innovation approach. Social Enterprise Journal, 13(3), 234-250. 